\title{
Plasma free 25-hydroxyvitamin D, vitamin D binding protein, and risk of breast cancer in the Nurses' Health Study II
}

\author{
Jun Wang, \\ Division of Biostatistics and Epidemiology, School of Public Health and Health Sciences, \\ University of Massachusetts Amherst, 715 N. Pleasant Street, Amherst, MA 01003, USA
}

\begin{abstract}
A. Heather Eliassen,
Channing Division of Network Medicine, Department of Medicine, Brigham and Women's Hospital and Harvard Medical School, 181 Longwood Avenue, Boston, MA 02115, USA. Department of Epidemiology, Harvard School of Public Health, 677 Huntington Avenue, Boston, MA 02115, USA

Donna Spiegelman,

Department of Epidemiology, Harvard School of Public Health, 677 Huntington Avenue, Boston, MA 02115, USA. Department of Biostatistics, Harvard School of Public Health, 677 Huntington Avenue, Boston, MA 02115, USA
\end{abstract}

Walter C. Willett, and

Channing Division of Network Medicine, Department of Medicine, Brigham and Women's Hospital and Harvard Medical School, 181 Longwood Avenue, Boston, MA 02115, USA. Department of Epidemiology, Harvard School of Public Health, 677 Huntington Avenue, Boston, MA 02115, USA. Department of Nutrition, Harvard School of Public Health, 677 Huntington Avenue, Boston, MA 02115, USA

\section{Susan E. Hankinson}

Division of Biostatistics and Epidemiology, School of Public Health and Health Sciences, University of Massachusetts Amherst, 715 N. Pleasant Street, Amherst, MA 01003, USA. Channing Division of Network Medicine, Department of Medicine, Brigham and Women's Hospital and Harvard Medical School, 181 Longwood Avenue, Boston, MA 02115, USA. Department of Epidemiology, Harvard School of Public Health, 677 Huntington Avenue, Boston, MA 02115, USA Jun Wang: junw@schoolph.umass.edu

\section{Abstract}

Purpose-Prior prospective studies, including our own, have evaluated total plasma 25hydroxyvitamin D [25(OH)D] and breast cancer risk with inconsistent results. However, recent studies suggest that some vitamin D functions may be more relevant to the unbound (free) fraction of 25(OH)D. Vitamin D binding protein (DBP) influences the free 25(OH)D levels and thus possibly the biological activities of vitamin D.

(C) Springer International Publishing Switzerland 2014

Correspondence to: Jun Wang, junw@schoolph. umass . edu.

Conflict of interest All authors disclosed no potential conflict of interest. 
Methods-We conducted a case-control study nested within the Nurses' Health Study II to evaluate the association of plasma free $25(\mathrm{OH}) \mathrm{D}$ and $\mathrm{DBP}$ with breast cancer risk in predominantly premenopausal women. Plasma samples were assayed for $25(\mathrm{OH}) \mathrm{D}$ and DBP in 584 case-control pairs. Free 25(OH)D levels were calculated based on plasma levels of total $25(\mathrm{OH}) \mathrm{D}, \mathrm{DBP}$, and a constant value representing average albumin levels. Conditional logistic regression was used to estimate relative risks (RRs) and $95 \%$ confidence intervals (CIs).

Results-We found no association between plasma calculated free $25(\mathrm{OH}) \mathrm{D}$ and risk of breast cancer overall (highest vs. lowest quartile RR $1.21,95 \%$ CI $0.83-1.77$, trend test $p$ value $=0.50$ ). No association was observed for plasma DBP as well (highest vs. lowest quartile RR 0.95, $95 \%$ CI 0.67-1.36, trend test $p$ value $=0.96$ ). Results were similar by tumor hormone receptor status. Neither the total nor the calculated free $25(\mathrm{OH}) \mathrm{D}$ and breast cancer association substantially varied by plasma DBP levels.

Conclusions-Our study does not support an important role of either calculated circulating free $25(\mathrm{OH}) \mathrm{D}$ or circulating DBP levels in breast cancer risk among predominantly premenopausal women.

\section{Keywords}

Free 25-hydroxyvitamin D; Vitamin D binding protein; Breast cancer; Epidemiology; Prospective

\section{Introduction}

Experimental studies indicate that vitamin D may have an important impact on both normal breast development and breast cancer [1]. Vitamin D, a steroid hormone, exerts most of its biological function through binding to the vitamin D receptor (VDR), which is expressed in normal mammary epithelial tissue. In animal models, knockout of VDR results in extensive ductal elongation and branching, as well as enhanced responsiveness to exogenous estrogen and progesterone [2]. Furthermore, substantial data suggest that vitamin D has antiproliferative, pro-apoptotic, and anti-invasion properties in breast cancer models [1]. However, in prospective epidemiologic studies, the association between circulating 25hydroxyvitamin $\mathrm{D}[25(\mathrm{OH}) \mathrm{D}]$, the main form of circulating vitamin $\mathrm{D}$ as well as the established biomarker of vitamin D status [3], and risk of breast cancer has been inconsistent [4-15]. One possible explanation for the conflicting results could be that current epidemiologic studies have only measured total circulating levels and have not differentiated the bound and unbound (free) fractions of vitamin D metabolites. According to the 'free hormone' hypothesis [16], unbound vitamin D metabolites are more likely to exert biological activity (i.e., entry through the cell membrane to bind to their intracellular receptors) than protein-bound vitamin D metabolites.

Approximately 85-90\% of circulating 25(OH)D and 1,25-dihydroxyvitamin $\mathrm{D}_{3}$ $\left[1,25(\mathrm{OH})_{2} \mathrm{D}_{3}\right]$, the biologically active form of vitamin $\mathrm{D}$ metabolite, are bound to vitamin D binding protein (DBP), and about $10-15 \%$ are loosely bound to albumin, leaving $<1 \%$ as the free form $[17,18]$. Consistent with the 'free hormone' hypothesis, several recent studies have shown that some functions of vitamin D may be more closely related to the free or bioavailable fraction of $25(\mathrm{OH}) \mathrm{D}$ than to total $25(\mathrm{OH}) \mathrm{D}$ levels. For instance, the free or 
bioavailable fraction (defined as free plus albumin-bound portion) of circulating $25(\mathrm{OH}) \mathrm{D}$ was more strongly associated with bone mineral density than the total levels in healthy adults [19] and patients with kidney diseases [20].

One of the major functions of DBP is the binding and transporting of vitamin D metabolites. Circulating DBP levels are approximately 20 times higher than the total circulating vitamin D levels [21]. Experimental studies show that DBP prolongs the half-life of serum 25(OH)D but can also inhibit the biological function of injected $1,25(\mathrm{OH})_{2} \mathrm{D}_{3}$ in mice [22]. In several recent nested case-control studies, circulating DBP levels modified the association between $25(\mathrm{OH}) \mathrm{D}$ and risk of several cancers, including bladder [23], pancreatic [24], and prostate [25], further suggesting a role of DBP in the etiology of cancer.

We previously found no association between plasma $25(\mathrm{OH}) \mathrm{D}$ and breast cancer risk in a study population of predominantly premenopausal women in the Nurses' Health Study II (NHS II) [12]. However, given the potentially important role of 'free' vitamin D in exerting its biological activities, in the current analysis, we examined the association between plasma calculated free $25(\mathrm{OH}) \mathrm{D}$ and breast cancer risk in the same study population. In light of the reported modifying role of DBP on 25(OH)D and risk of several cancers, we also assessed whether there was a direct association between circulating DBP and breast cancer risk and whether the $25(\mathrm{OH}) \mathrm{D}$ and breast cancer association varied by DBP levels.

\section{Materials and methods}

\section{Study population}

The NHSII was established in 1989, when 116,430 female registered nurses, aged 25-42 years, completed, and returned an initial questionnaire. The majority of the participants were white ( $94 \%)$ and approximately $2 \%$ each for African American, Asian, and Hispanic. The cohort has been followed biennially by mailed questionnaires to update exposures and to ascertain newly diagnosed disease.

Between 1996 and 1999, 29,611 cohort members who were cancer-free and between 32 and 54 years old provided a blood sample in response to having been sent a blood collection kit and a short questionnaire. Details of this cohort and blood collection have been described previously [26]. Briefly, 18,521 premenopausal women who had not used exogenous hormones (i.e., postmenopausal hormonal therapy or oral contraceptives) or been pregnant in the past 6 months provided an initial blood sample drawn on the third to fifth day of the menstrual cycle (follicular blood collection) and a second sample drawn 7-9 days before the anticipated start of the next cycle (luteal blood collection). Participants were sent a blood collection kit and a short questionnaire. Follicular plasma was aliquoted by the participants and frozen in their home until the second blood collection. Participants shipped both follicular plasma and luteal whole blood samples via overnight courier with an ice pack, to the laboratory where luteal blood samples were processed and separated into plasma, red blood cell, and white blood cell components, and aliquoted into labeled cryotubes. Women who were not eligible to provide timed blood samples (e.g., perimenopausal or postmenopausal) or declined to provide timed samples gave a single blood sample. These samples were shipped and processed similarly to luteal samples. All samples have been 
stored in liquid nitrogen freezers (less than $-130{ }^{\circ} \mathrm{C}$ ) since collection. Characteristics of women who donated blood samples (e.g., age, BMI) were similar to the entire NHSII cohort [26]. The study was approved by the Committee on the Use of Human Subjects in Research at the Brigham and Women's Hospital and the Harvard School of Public Health.

\section{Case identification and control selection}

Details of the case ascertainment and control selection have been described elsewhere [12]. In brief, breast cancer cases were identified through the biennial questionnaires: Cases had no previously reported cancer diagnosis before blood collection and were diagnosed after blood collection but before June 1, 2007. A total of 610 cases who donated blood and participated in the previous study of plasma $25(\mathrm{OH}) \mathrm{D}$ were included [12], with mean time from blood drawn to diagnosis of 57 months (range 1-127 months). Cases were confirmed by either medical record review (95\%) or verbal confirmation by the nurse (5\%). The latter cases were included because of the $>99 \%$ concordance rate between medical record review and self-report. Although the previous study of plasma 25(OH)D included 2 controls per case, due to financial constraints, only one control was matched to each case in the current analysis ( $n=610$ controls) by age ( \pm 2 years); race/ethnicity (Caucasian, African American, Hispanic, Asian, other); menopausal status at blood collection and diagnosis (premenopausal, postmenopausal, unknown/missing); month and year of blood drawn ( \pm 2 months); luteal day (for timed samples; \pm 1 day); time of day $( \pm 2 \mathrm{~h})$; and fasting status $(<2$, $2-4,5-7,8-11,>12$ h).

\section{Laboratory assays}

Details of the plasma 25(OH)D measurements have been described previously [12]. Briefly, plasma $25(\mathrm{OH}) \mathrm{D}$ was assayed using a radioimmunoassay with radioiodinated tracers after acetonitrile extraction at Heartland Assays Inc. (Ames, IA, U.S.). Assays were run in two batches; the overall coefficients of variation (CVs) from blinded replicate quality control samples in each batch were 10.7 and $6.0 \%$. Plasma DBP was assayed in the laboratory of Dr. Nader Rifai (Children's Hospital, Boston, MA) using an enzyme-linked immunosorbent assay (ELISA) from R\&D systems (Minneapolis, MN). All samples for DBP were assayed in a single batch, and the $\mathrm{CV}$ from blinded replicate quality control samples was $6.7 \%$. Case-control pairs were assayed together, and laboratory personnel were blinded to case, control, or quality control status. Of the 610 pairs, 26 pairs were not sent to the laboratory because of insufficient plasma remaining for DBP assays, leaving a total of 584 case-control sets in the final analysis.

\section{Covariate data}

Demographic and breast cancer risk factor information was obtained from the biennial NHS II questionnaires, and a questionnaire completed at the time of the blood drawn. Age at menarche, weight at age 18, and height were queried in the baseline questionnaire in 1989. Family history of breast cancer was ascertained in 1989 and 1997. Parity, age at first birth, diagnosis of benign breast disease, alcohol consumption, menopausal status, weight, and oral contraceptive use were assessed biennially. Weight and alcohol consumption were also 
asked at the time of blood collection. For covariates with multiple assessments, information from the questionnaire completed closest to the blood collection was used.

\section{Statistical analysis}

We first assessed statistical outliers using the generalized extreme studentized deviate manyoutlier detection approach [27]. Five participants with low 25(OH)D values ( $₫ 10.7 \mathrm{nmol} / \mathrm{L}$ ) were identified; however, these low values were likely to be true because these participants had very low vitamin D dietary intake (i.e., 50-100 IU/day) and donated blood during the winter, and thus, these values were retained in the analysis. Only one participant who had an outlier value at the low end of DBP $(837.1 \mathrm{nmol} / \mathrm{L})$ was identified. However, results were materially unchanged by excluding this outlier; thus, we kept this participant in the analyses.

Plasma-free 25(OH)D was calculated by the following equation [18]:

$$
\text { Free } 25(\mathrm{OH}) \mathrm{D}=\frac{\operatorname{Total} 25(\mathrm{OH}) \mathrm{D}}{1+\left(\left[6 \times 10^{5}\right] \times\left[5.8 \times 10^{-6}\right]\right)+\left(\left[7 \times 10^{8}\right] \times \mathrm{DBP}\right)}
$$

where $6 \times 10^{5}$ and $7 \times 10^{8}$ represent the affinity constants of $25(\mathrm{OH}) \mathrm{D}$ to albumin and DBP, respectively, and all concentrations are expressed in mol/L. Calculated circulating free $25(\mathrm{OH}) \mathrm{D}$ using this equation and measured free $25(\mathrm{OH}) \mathrm{D}$ using centrifugal ultrafiltration were reported to be highly correlated $(r=0.9)$ [18]. Plasma albumin was not measured in our study; instead, a constant $\left(5.8 \times 10^{-6} \mathrm{~mol} / \mathrm{L}\right)$ was used in the analysis based on previous studies [28] given that only a small proportion (10-15\%) of circulating $25(\mathrm{OH}) \mathrm{D}$ binds to albumin and the inter-individual variation of albumin is small [28]. Thus, the quantitative impact of albumin on circulating free $25(\mathrm{OH}) \mathrm{D}$ levels is unsubstantial. For instance, for a given free $25(\mathrm{OH}) \mathrm{D}$ value of $18.96 \mathrm{pmol} / \mathrm{L}$ calculated with albumin as $5.8 \times 10^{-6} \mathrm{~mol} / \mathrm{L}$, this value remained the same when using albumin levels of either $4.6 \times 10^{-6} \mathrm{~mol} / \mathrm{L}$ or $7.3 \times$ $10^{-6} \mathrm{~mol} / \mathrm{L}$, levels representing the normal lower and upper range of circulating albumin. Of note, the ratio of total $25(\mathrm{OH}) \mathrm{D}$ to $\mathrm{DBP}$ has previously been used as an index of free 25(OH)D [29]. Without measuring circulating albumin levels, the molar ratio and calculated free $25(\mathrm{OH}) \mathrm{D}$ levels are perfectly correlated and only differ by the albumin constant.

For the primary analysis, conditional logistic regression was used to estimate relative risks (RRs) and $95 \%$ confidence intervals (CIs). Quartile cutoff points of total 25(OH)D and calculated free 25(OH)D were defined in two ways: The first set was determined based on the distribution among all controls; the second set was based on season-specific cutoff points based on blood collection dates among the controls: February to April, May to July, August to October, and November to January. Quartile cutoff points of DBP, which does not vary by season, were determined based on the distribution among all controls. Multivariate models were adjusted for body mass index (BMI) at age 18 and at blood collection, age at menarche, parity and age at first birth, history of benign breast disease, family history of breast cancer, and alcohol consumption (see Tables 2, 3, 4 footnote for exposure categories). Trend tests were conducted by modeling quartile medians and calculating the Wald statistic. The dose-response relationship was assessed using restricted cubic spline models [30, 31]. Tests of interaction between plasma total, free $25(\mathrm{OH}) \mathrm{D}$ or DBP and a potential modifying 
variable were performed using the likelihood ratio test by comparing models with and without a cross-product term of the quartile medians of each vitamin D variable and the modifying variable. In stratified analyses, unconditional logistic regression adjusted for the matching factors and the covariates given above was used to maximize statistical power. Results from the multivariable unconditional and conditional logistic regression were very similar. In stratified analyses by tumor subtype (i.e., ER-/PR-), we collapsed several categories of alcohol consumption due to small numbers of cases or controls in some categories.

To test whether the association between calculated free 25(OH)D or DBP and breast cancer risk differed by estrogen receptor (ER) or progesterone receptor (PR) status of the tumor, we used polytomous unconditional logistic regression [32]. Because there were few cases with $\mathrm{ER}+/ \mathrm{PR}-$ or $\mathrm{ER}-/ \mathrm{PR}+$, tests of disease heterogeneity were performed primarily for $\mathrm{ER}+/ \mathrm{PR}$ + versus ER-/PR-. A likelihood ratio test comparing a model with a common slope for calculated free 25(OH)D or DBP with a model with separate slopes for each case group was used. All $p$ values were based on two-sided tests and considered statistically significant if $<0.05$. All analyses were performed using SAS software version 9.2 (SAS Institute, Cary, NC, USA).

\section{Results}

Cases were more likely to have a family history of breast cancer and a history of benign breast disease than controls (Table 1). BMI at age 18 and at blood collection was slightly higher in controls than in cases. Plasma levels of calculated free 25(OH)D and DBP were similar in cases and controls.

Calculated free 25(OH)D was highly correlated with total 25(OH)D (Spearman's correlation coefficient $[\mathrm{r}]$ among controls $=0.76, p<0.0001)$ and inversely correlated with DBP $(r=$ -0.51 among controls, $p<0.0001)$. In addition, among controls, free $25(\mathrm{OH}) \mathrm{D}$ was inversely correlated with BMI at blood drawn $(r=-0.29, p<0.0001)$ while positively correlated with alcohol consumption $(r=0.19, p<0.0001)$. The high correlation between total and calculated free 25(OH)D remained after controlling for age, BMI at blood collection, and alcohol consumption $(r=0.73, p<0.0001)$. DBP was not correlated with age, BMI, smoking, or alcohol consumption.

As expected, plasma total 25(OH)D was not associated with risk of breast cancer overall in this analysis (the highest versus lowest quartile RR $1.18,95 \%$ CI 0.80-1.76; trend test $p$ value $=0.49$ ), and the estimates were very similar to the results in our prior report (top vs. bottom quartile RR 1.20, $95 \%$ CI 0.88-1.63; trend test $p$ value $=0.32$ ) [12]. Calculated free $25(\mathrm{OH}) \mathrm{D}$ was not significantly associated with breast cancer overall (the highest vs. lowest quartile RR $1.21,95 \%$ CI $0.83-1.77$; trend test $p$ value $=0.50$, Table 2 ). Further analyses by tumor subtypes did not show significant differences. The effect estimates were similar in invasive cases (the highest vs. lowest quartile RR $1.15,95 \%$ CI $0.77-1.72$; trend test $p$ value $=0.69$ ). When stratified by tumor hormone receptor status, no significant heterogeneity was found (heterogeneity test $p$ value for $\mathrm{ER}+/ \mathrm{PR}+\mathrm{vs}$. ER-/PR- = 0.12). There was no significant linear or nonlinear association detected when using restricted cubic 
spline models. Calculated free 25(OH)D levels were significantly higher in the months of May to October than in the months of November to April ( $p<0.0001$, data not shown). When stratifying by season of blood drawn (i.e., May-July, August-October, NovemberJanuary, February-April), we found an overall null association in each of the strata. A nonsignificant positive association was observed for months with more light (i.e., May to July, the highest vs. lowest RR $1.26,95 \%$ CI $0.62-2.56$, trend test $p$ value $=0.42$ ) as well as for darker months (i.e., November to January, the highest vs. lowest RR 1.43, 95 \% CI 0.663.11 ; trend test $p$ value $=0.42$ ). We further performed analyses using season-specific cutoff points for calculated free $25(\mathrm{OH}) \mathrm{D}$, and the results remained very similar to those without using season-specific cutoffs (data not shown).

Plasma DBP was not associated with breast cancer overall (the highest vs. lowest quartile RR $0.95,95 \%$ CI 0.67-1.36; trend test $p$ value $=0.96$, Table 3 ) or invasive cases (the highest vs. lowest quartile RR $1.1895 \%$ CI $0.82-1.71$; trend test $p$ value $=0.25$ ) in our study population. Further, no significant heterogeneity was observed by hormone receptor subtype (heterogeneity test $p$ value for $\mathrm{ER}+/ \mathrm{PR}+\mathrm{vs}$. ER-/PR- = 0.78). No significant linear or nonlinear association was detected for DBP as well using the restricted cubic spline models.

When we stratified by the median level of plasma DBP, we did not observe any significant variation in the associations of either total or calculated free $25(\mathrm{OH}) \mathrm{D}$ with breast cancer risk (interaction test $p$ value $=0.75$ and 0.82 , respectively; Table 4). We also tested whether BMI at blood collection ( $<25 \mathrm{vs} . \quad 25 \mathrm{~kg} / \mathrm{m}^{2}$ ) modified the association between calculated free $25(\mathrm{OH}) \mathrm{D}$ or DBP and breast cancer, and observed a significant interaction for free $25(\mathrm{OH}) \mathrm{D}$ (interaction test $p$ value $<0.0001$ ) but not for DBP (interaction test $p$ value $=0.75$ ). There was an increasing risk associated with increased calculated free $25(\mathrm{OH}) \mathrm{D}$ levels among women with BMI $\geq 2 \mathrm{~kg} / \mathrm{m}^{2}$ (trend test $p$ value $=0.01$ ), with a more than doubled risk observed in the highest versus lowest quartile $(\mathrm{OR}=2.11,95 \% \mathrm{CI} 1.21-3.70)$. No significant association was found among women with BMI $<25 \mathrm{~kg} / \mathrm{m}^{2}$.

\section{Discussion}

To our knowledge, this is the first investigation of the association between circulating free $25(\mathrm{OH}) \mathrm{D}, \mathrm{DBP}$, and risk of breast cancer. We observed no association between plasma calculated free $25(\mathrm{OH}) \mathrm{D}$ and risk of breast cancer overall or according to tumor invasiveness or hormone receptor status. Similarly, we did not find an association between plasma DBP and risk of breast cancer overall or by tumor subtype. Our data also suggest no significant interaction between circulating DBP and either total or calculated free $25(\mathrm{OH}) \mathrm{D}$ on risk of breast cancer overall. The significant interaction between calculated free 25(OH)D and BMI was expected, given a similar interaction was observed for total 25(OH)D in our previous study [12] and the high correlation between total and calculated free $25(\mathrm{OH}) \mathrm{D}(r=$ 0.76 among controls).

Although an overall null association was found for plasma total $25(\mathrm{OH}) \mathrm{D}$ and breast cancer in this and in our previous study [12], both of which consisted of predominantly premenopausal women, an earlier study reported a suggestive inverse association in 
predominantly postmenopausal women in the NHS (RR for highest vs. lowest quintile $=$ $0.73,95 \%$ CI 0.49-1.07; trend test $p$ value $=0.06$ ) [4]. Consistent with this apparent differential effect of plasma total $25(\mathrm{OH}) \mathrm{D}$ on breast cancer by menopausal status, a recent dose-response meta-analysis also suggested a possible nonlinear inverse association in postmenopausal but not premenopausal women [33]. However, the mechanisms underlying a potential difference by menopausal status are not known.

Although no studies to date have evaluated the effect of circulating free $25(\mathrm{OH}) \mathrm{D}$ on risk of breast cancer, several prospective studies have assessed the association between the molar ratio of 25(OH)D to DBP, a proxy for free circulating 25(OH)D [29], and risk of cancers of different origins. Given that we did not measure albumin levels in our study, the calculated $25(\mathrm{OH}) \mathrm{D}$ based on a constant value representing average albumin levels is statistically identical to the molar ratio. In case-control studies nested within the Alpha-Tocopherol Beta-Carotene (ATBC) Cancer Prevention study cohort, the molar ratio of 25(OH)D to DBP was positively associated with risk of pancreatic cancer (RR for the highest vs. the lowest quartile $=1.86,95 \%$ CI 0.97-3.56; trend test $p$ value $=0.04)[24]$ and prostate cancer $(\mathrm{RR}$ for the highest vs. the lowest quintile $=1.36,95 \% \mathrm{CI} 0.97-1.91$; trend test $p$ value $=0.04$ ) [25] and suggestively associated with renal cell carcinoma (RR for the highest vs. lowest quartile $=1.61,95 \%$ CI 0.95-2.73; trend test $p$ value $=0.09$ ) [34]. However, in another case-control study nested within the ATBC study cohort, a non-significant inverse association was reported for bladder cancer [23], although results from a nested case-control study within the prostate, lung, colorectal, and ovarian (PLCO) cancer screening trial showed no association for bladder cancer [35]. In contrast to the positive association with prostate cancer found in the ATBC study, no association was observed in a nested casecontrol study in the Physicians' Health Study [36].

Several nested case-control studies including those mentioned above have examined the effects of circulating DBP on risk of several types of cancer. The findings were generally null for both bladder [23, 35] and prostate cancers [25, 36, 37], while inverse associations were reported for pancreatic cancer (RR for the highest vs. the lowest quintile $=0.67,95 \%$ CI 0.37-1.19; trend test $p$ value $=0.02$ ) [24] and renal cell carcinoma (RR for the top vs. bottom quartile $=0.17,95 \%$ CI 0.08-0.33; trend test $p$ value $<0.0001$ ) [34]. Despite the lack of a consistent overall association of DBP on risk of cancer at different sites, several analyses have suggested that the $25(\mathrm{OH}) \mathrm{D}$ - cancer association may vary by level of DBP [23-25]. The inverse 25(OH)D—bladder cancer [23] and the positive 25(OH)D—pancreatic cancer [24] associations were stronger among those with lower circulating DBP levels (below the median), suggesting that the 'free' fraction of 25(OH)D may be particularly relevant to the etiology of these cancers. However, in contrast, the positive $25(\mathrm{OH}) \mathrm{D}$ prostate cancer association was stronger among those with higher circulating DBP levels [25].

Overall, the current literature on the association between circulating 'free' or the proxy for 'free' $25(\mathrm{OH}) \mathrm{D}, \mathrm{DBP}$, and risk of cancer is limited and consists largely of results from the ATBC study. Differences in either the study population (e.g., ATBC participants were male smokers while $\sim 10 \%$ of our female participants smoked) or in the etiology of the cancers examined might partly explain the differing results. Of note, the correlation between 
circulating total and calculated free $25(\mathrm{OH})$ in our study and one of the nested case-control studies in the ATBC trial [24] were very similar ( $r=0.72$ in ATBC and $r=0.76$ in ours). Despite the relatively high correlation, total $25(\mathrm{OH}) \mathrm{D}$ levels only accounted for $52-58 \%$ $\left(r^{2}\right)$ of the variation in free levels based on these two studies. Future studies with larger sample sizes are needed to provide further insights of the relationship between the free portion and the total of $25(\mathrm{OH}) \mathrm{D}$.

In addition, contrary to the 'free hormone hypothesis,' recent experimental data have shown the existence of cellular uptake pathways for the DBP-bound vitamin D complex by the plasma membrane receptor megalin. Through this mechanism, carrier-bound vitamin D metabolites also may enter into the cell and be internalized via megalin-mediated endocytosis [38], thus potentially contributing to the activation of the VDR pathway [39]. Megalin is expressed in human mammary epithelial cell lines as well as some breast cancer cell lines [39]. Megalin-mediated cellular uptake of the DBP-bound vitamin D complex has been shown in several epithelial tissues, including the mammary gland [39, 40], but has not yet been reported in pancreas, bladder, or prostate tissues. Further epidemiologic studies of these associations as well as laboratory studies to further determine the presence and role of megalin are needed.

The major strengths in our study include the prospective collection of blood samples, relatively large study population, and low technical assay error (CV ranges 6-10\%). One limitation is that plasma $25(\mathrm{OH}) \mathrm{D}$ and DBP were measured at one point in time, although we have shown that a single measurement of $25(\mathrm{OH}) \mathrm{D}$ reliably reflects levels over at least a 3 -year period in the NHS (intraclass correlation coefficient $=0.72$ ) [41]. Circulating DBP levels maintain stable and do not vary substantially by season [29]. Another limitation is that we did not measure individual albumin levels; instead, a fixed value has been assigned to individuals. However, as mentioned above, it is unlikely to change the results given the negligible impact of circulating albumin levels on free 25(OH)D levels.

In conclusion, findings from our prospective study indicate no substantial impact of plasma calculated unbound 25(OH)D on risk of breast cancer. Further, we found no evidence of an important role of DBP in the vitamin D and breast cancer association in predominately premenopausal women.

\section{Acknowledgments}

This study was supported by Research Grants CA67262 and CA50385 from the National Cancer Institute. We would like to thank the participants and staff of the Nurses' Health Study II for their valuable contributions as well as the following state cancer registries for their help: AL, AZ, AR, CA, CO, CT, DE, FL, GA, ID, IL, IN, IA, KY, LA, ME, MD, MA, MI, NE, NH, NJ, NY, NC, ND, OH, OK, OR, PA, RI, SC, TN, TX, VA, WA, WY. The authors assume full responsibility for analyses and interpretation of these data.

\section{Abbreviations}

25(OH)D

DBP

VDR
25-Hydroxyvitamin D

Vitamin D binding protein

Vitamin D receptor 


$\begin{array}{ll}\mathbf{1 , 2 5}(\mathrm{OH})_{2} \mathbf{D}_{3} & 1,25 \text {-Dihydroxyvitamin } \mathrm{D}_{3} \\ \text { NHS } & \text { Nurses' Health Study } \\ \text { CV } & \text { Coefficient of variation } \\ \text { ER } & \text { Estrogen receptor } \\ \text { PR } & \text { Progesterone receptor } \\ \text { BMI } & \text { Body mass index } \\ \text { RR } & \text { Relative risk } \\ \text { CI } & \text { Confidence interval }\end{array}$

\section{References}

1. Lopes N, Paredes J, Costa JL, Ylstra B, Schmitt F. Vitamin D and the mammary gland: a review on its role in normal development and breast cancer. Breast Cancer Res. 2012; 14:211. [PubMed: 22676419]

2. Zinser G, Packman K, Welsh J. Vitamin D(3) receptor ablation alters mammary gland morphogenesis. Development. 2002; 129:3067-3076. [PubMed: 12070083]

3. Zerwekh JE. Blood biomarkers of vitamin D status. Am J Clin Nutr. 2008; 87:1087S-1091S. [PubMed: 18400739]

4. Bertone-Johnson ER, Chen WY, Holick MF, et al. Plasma 25-hydroxyvitamin D and 1,25dihydroxyvitamin D and risk of breast cancer. Cancer Epidemiol Biomarkers Prev. 2005; 14:19911997. [PubMed: 16103450]

5. Chlebowski RT, Johnson KC, Kooperberg C, et al. Calcium plus vitamin D supplementation and the risk of breast cancer. J Natl Cancer Inst. 2008; 100:1581-1591. [PubMed: 19001601]

6. Freedman DM, Chang SC, Falk RT, et al. Serum levels of vitamin D metabolites and breast cancer risk in the prostate, lung, colorectal, and ovarian cancer screening trial. Cancer Epidemiol Biomarkers Prev. 2008; 17:889-894. [PubMed: 18381472]

7. McCullough ML, Stevens VL, Patel R, et al. Serum 25-hydroxyvitamin D concentrations and postmenopausal breast cancer risk: a nested case control study in the Cancer Prevention Study-II Nutrition Cohort. Breast Cancer Res. 2009; 11:R64. [PubMed: 19715600]

8. Rejnmark L, Tietze A, Vestergaard P, et al. Reduced prediagnostic 25-hydroxyvitamin D levels in women with breast cancer: a nested case-control study. Cancer Epidemiol Biomarkers Prev. 2009; 18:2655-2660. [PubMed: 19789365]

9. Agborsangaya CB, Surcel HM, Toriola AT, et al. Serum 25-hydroxyvitamin D at pregnancy and risk of breast cancer in a prospective study. Eur J Cancer. 2010; 46:467-470. [PubMed: 20022237]

10. Almquist M, Bondeson AG, Bondeson L, Malm J, Manjer J. Serum levels of vitamin D, PTH and calcium and breast cancer risk-a prospective nested case-control study. Int J Cancer. 2010; 127:2159-2168. [PubMed: 20112341]

11. Engel P, Fagherazzi G, Boutten A, et al. Serum 25(OH) vitamin D and risk of breast cancer: a nested case-control study from the French E3 N cohort. Cancer Epidemiol Biomarkers Prev. 2010; 19:2341-2350. [PubMed: 20826834]

12. Eliassen AH, Spiegelman D, Hollis BW, Horst RL, Willett WC, Hankinson SE. Plasma 25hydroxyvitamin D and risk of breast cancer in the Nurses' Health Study II. Breast Cancer Res. 2011; 13:R50. [PubMed: 21569367]

13. Kuhn T, Kaaks R, Becker S, et al. Plasma 25-hydroxyvi-tamin D and the risk of breast cancer in the European prospective investigation into cancer and nutrition: a nested case-control study. Int J Cancer. 2013; 133:1689-1700. [PubMed: 23526380] 
14. Scarmo S, Afanasyeva Y, Lenner P, et al. Circulating levels of 25-hydroxyvitamin D and risk of breast cancer: a nested case-control study. Breast Cancer Res. 2013; 15:R15. [PubMed: 23442740]

15. Mohr SB, Gorham ED, Alcaraz JE, et al. Serum 25-hy-droxyvitamin D and breast cancer in the military: a case-control study utilizing pre-diagnostic serum. Cancer Causes Control. 2013; 24:495-504. [PubMed: 23296455]

16. Mendel CM. The free hormone hypothesis: a physiologically based mathematical model. Endocr Rev. 1989; 10:232-274. [PubMed: 2673754]

17. Bikle DD, Siiteri PK, Ryzen E, Haddad JG. Serum protein binding of 1,25-dihydroxyvitamin D: a reevaluation by direct measurement of free metabolite levels. J Clin Endocrinol Metab. 1985; 61:969-975. [PubMed: 3840175]

18. Bikle DD, Gee E, Halloran B, Kowalski MA, Ryzen E, Haddad JG. Assessment of the free fraction of 25-hydroxyvitamin $\mathrm{D}$ in serum and its regulation by albumin and the vitamin D-binding protein. J Clin Endocrinol Metab. 1986; 63:954-959. [PubMed: 3745408]

19. Powe CE, Ricciardi C, Berg AH, et al. Vitamin D-binding protein modifies the vitamin D-bone mineral density relationship. J Bone Miner Res. 2011; 26:1609-1616. [PubMed: 21416506]

20. Bhan I, Powe CE, Berg AH, et al. Bioavailable vitamin D is more tightly linked to mineral metabolism than total vitamin D in incident hemodialysis patients. Kidney Int. 2012; 82:84-89. [PubMed: 22398410]

21. White P, Cooke N. The multifunctional properties and characteristics of vitamin D-binding protein. Trends Endocrinol Metab. 2000; 11:320-327. [PubMed: 10996527]

22. Safadi FF, Thornton $\mathrm{P}$, Magiera H, et al. Osteopathy and resistance to vitamin D toxicity in mice null for vitamin D binding protein. J Clin Invest. 1999; 103:239-251. [PubMed: 9916136]

23. Mondul AM, Weinstein SJ, Virtamo J, Albanes D. Influence of vitamin D binding protein on the association between circulating vitamin D and risk of bladder cancer. Br J Cancer. 2012; 107:1589-1594. [PubMed: 22990651]

24. Weinstein SJ, Stolzenberg-Solomon RZ, Kopp W, Rager H, Virtamo J, Albanes D. Impact of circulating vitamin $\mathrm{D}$ binding protein levels on the association between 25 -hydrox-yvitamin $\mathrm{D}$ and pancreatic cancer risk: a nested case-control study. Cancer Res. 2012; 72:1190-1198. [PubMed: 22232734]

25. Weinstein SJ, Mondul AM, Kopp W, Rager H, Virtamo J, Albanes D. Circulating 25hydroxyvitamin D, vitamin D-binding protein and risk of prostate cancer. Int J Cancer. 2013; 132:2940-2947. [PubMed: 23180681]

26. Tworoger SS, Sluss P, Hankinson SE. Association between plasma prolactin concentrations and risk of breast cancer among predominately premenopausal women. Cancer Res. 2006; 66:24762482. [PubMed: 16489055]

27. Rosner B. Percentage points for a generalized ESD many-outlier procedure. Technometrics. 1983; 25:165-172.

28. Manolio TA, Burke GL, Psaty BM, et al. Black-white differences in subclinical cardiovascular disease among older adults: the Cardiovascular Health Study. CHS Collaborative Research Group. J Clin Epidemiol. 1995; 48:1141-1152. [PubMed: 7636516]

29. Bouillon R, Van Assche FA, Van Baelen H, Heyns W, De Moor P. Influence of the vitamin Dbinding protein on the serum concentration of 1,25-dihydroxyvitamin D3. Significance of the free 1,25-dihydroxyvitamin D3 concentration. J Clin Invest. 1981; 67:589-596. [PubMed: 6894152]

30. Durrleman S, Simon R. Flexible regression models with cubic splines. Stat Med. 1989; 8:551-561. [PubMed: 2657958]

31. Govindarajulu US, Spiegelman D, Thurston SW, Ganguli B, Eisen EA. Comparing smoothing techniques in Cox models for exposure-response relationships. Stat Med. 2007; 26:3735-3752. [PubMed: 17538974]

32. Marshall RJ, Chisholm EM. Hypothesis testing in the polychotomous logistic model with an application to detecting gastrointestinal cancer. Stat Med. 1985; 4:337-344. [PubMed: 4059720]

33. Bauer SR, Hankinson SE, Bertone-Johnson ER, Ding EL. Plasma vitamin D levels, menopause, and risk of breast cancer: dose-response meta-analysis of prospective studies. Medicine. 2013; 92:123-131. [PubMed: 23625163] 
34. Mondul AM, Weinstein SJ, Moy KA, Mannisto S, Albanes D. Vitamin D binding protein, circulating vitamin D, and risk of renal cell carcinoma. Int J Cancer. 201310.1002/ijc.28596

35. Mondul AM, Weinstein SJ, Horst RL, Purdue M, Albanes D. Serum vitamin D and risk of bladder cancer in the Prostate, Lung, Colorectal, and Ovarian (PLCO) Cancer Screening trial. Cancer Epidemiol Biomarkers Prev. 2012; 21:1222-1225. [PubMed: 22623707]

36. Gann PH, Ma J, Hennekens CH, Hollis BW, Haddad JG, Stampfer MJ. Circulating vitamin D metabolites in relation to subsequent development of prostate cancer. Cancer Epidemiol Biomarkers Prev. 1996; 5:121-126. [PubMed: 8850273]

37. Corder EH, Friedman GD, Vogelman JH, Orentreich N. Seasonal variation in vitamin D, vitamin D-binding protein, and dehydroepiandrosterone: risk of prostate cancer in black and white men. Cancer Epidemiol Biomarkers Prev. 1995; 4:655-659. [PubMed: 8547833]

38. Willnow TE, Nykjaer A. Cellular uptake of steroid carrier proteins-mechanisms and implications. Mol Cell Endocrinol. 2010; 316:93-102. [PubMed: 19646505]

39. Rowling MJ, Kemmis CM, Taffany DA, Welsh J. Megalin-mediated endocytosis of vitamin D binding protein correlates with 25-hydroxycholecalciferol actions in human mammary cells. J Nutr. 2006; 136:2754-2759. [PubMed: 17056796]

40. Chlon TM, Taffany DA, Welsh J, Rowling MJ. Retinoids modulate expression of the endocytic partners megalin, cubilin, and disabled-2 and uptake of vitamin D-binding protein in human mammary cells. J Nutr. 2008; 138:1323-1328. [PubMed: 18567755]

41. Kotsopoulos J, Tworoger SS, Campos H, et al. Reproducibility of plasma and urine biomarkers among premenopausal and postmenopausal women from the Nurses' Health Studies. Cancer Epidemiol Biomarkers Prev. 2010; 19:938-946. [PubMed: 20332276] 
Table 1

Characteristics of breast cancer cases and controls in the NHS II ${ }^{a}$

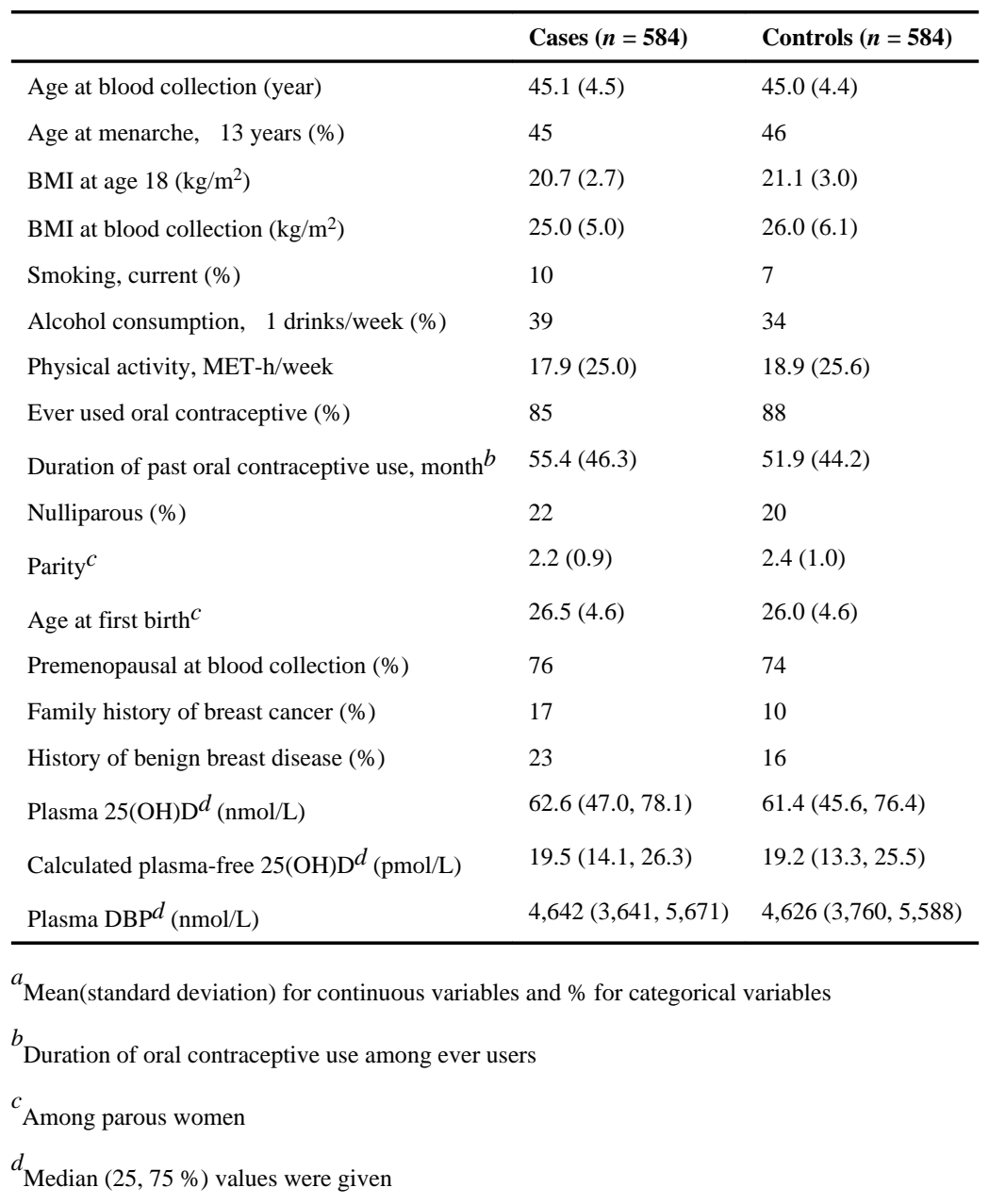




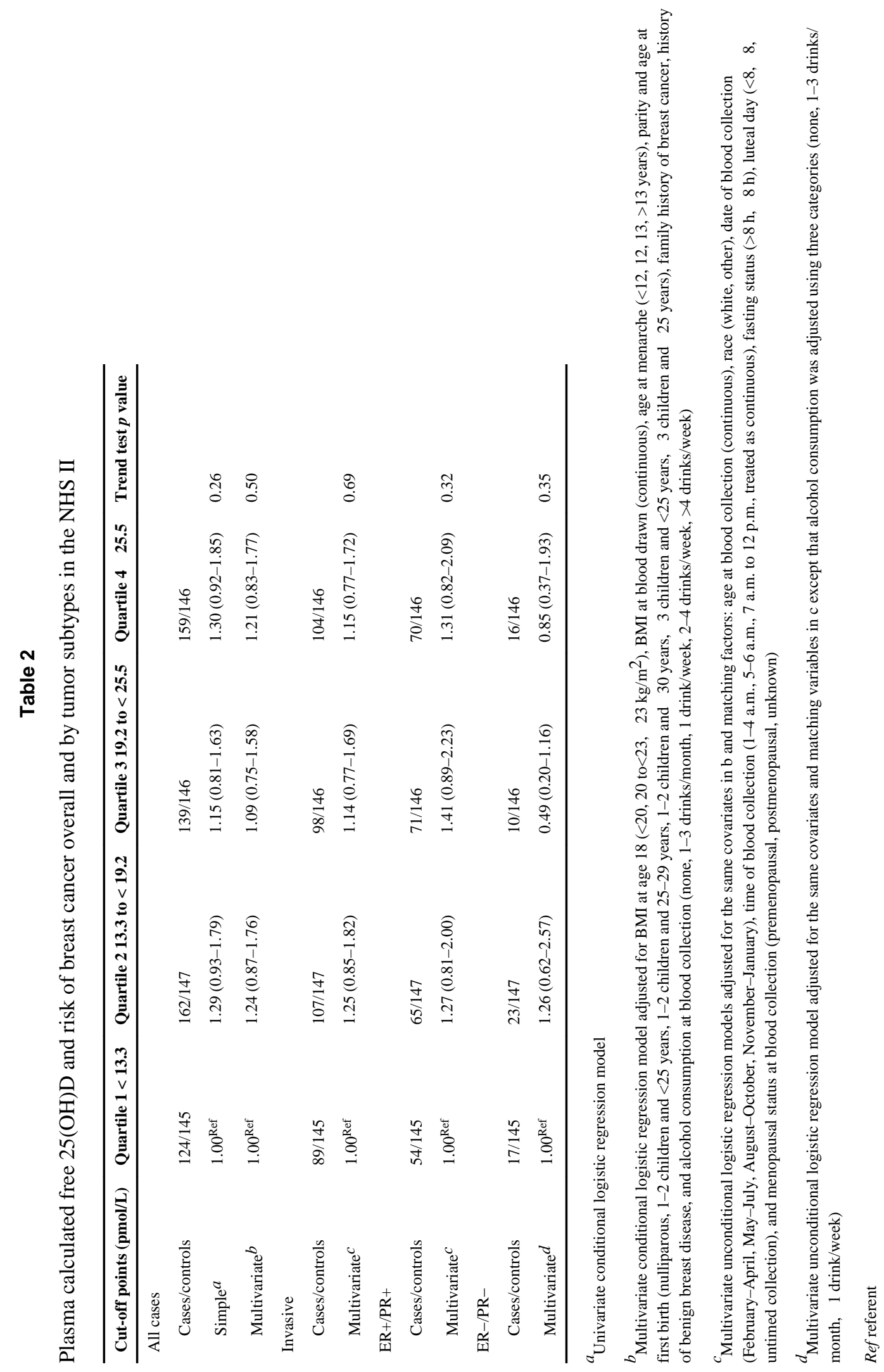




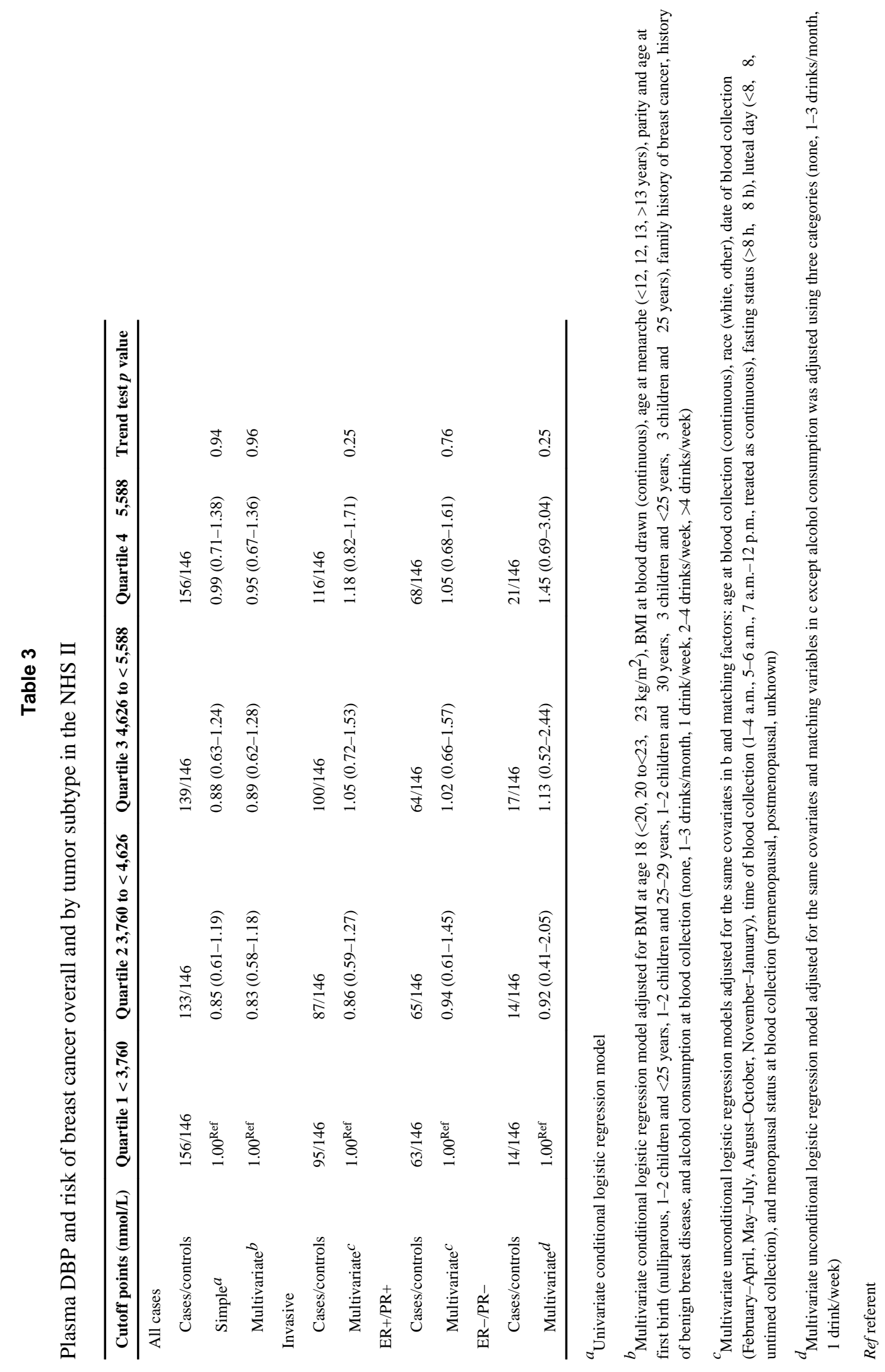




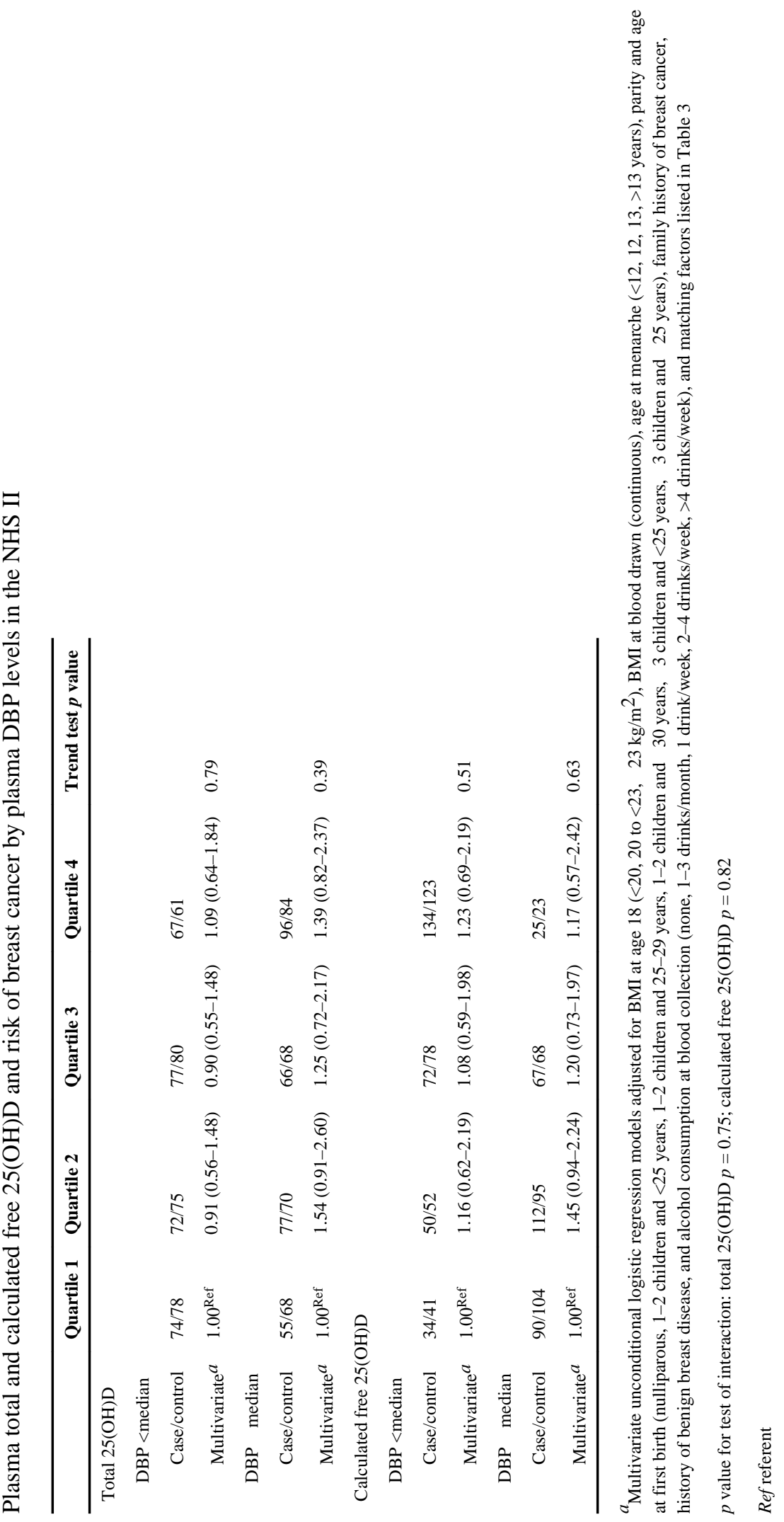

